\title{
Mondor's Disease
}

\section{Rare case of a painful breast lump in a middle-aged woman}

*Philips G. Michael, Tahra Al-Saadi, Rakesh Jamkhandikar

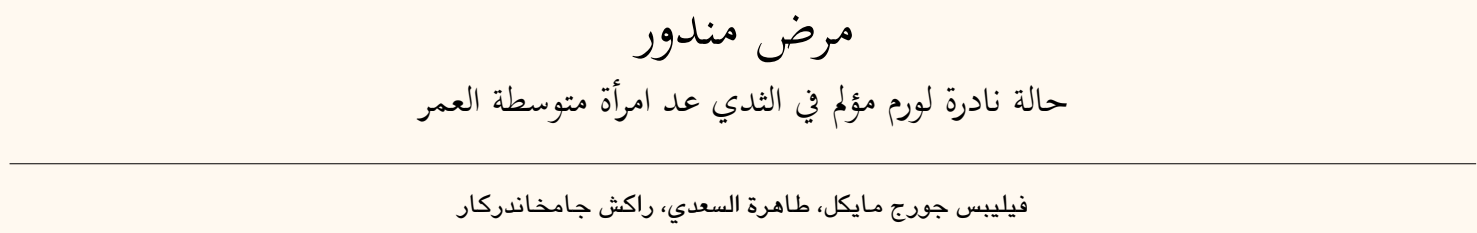
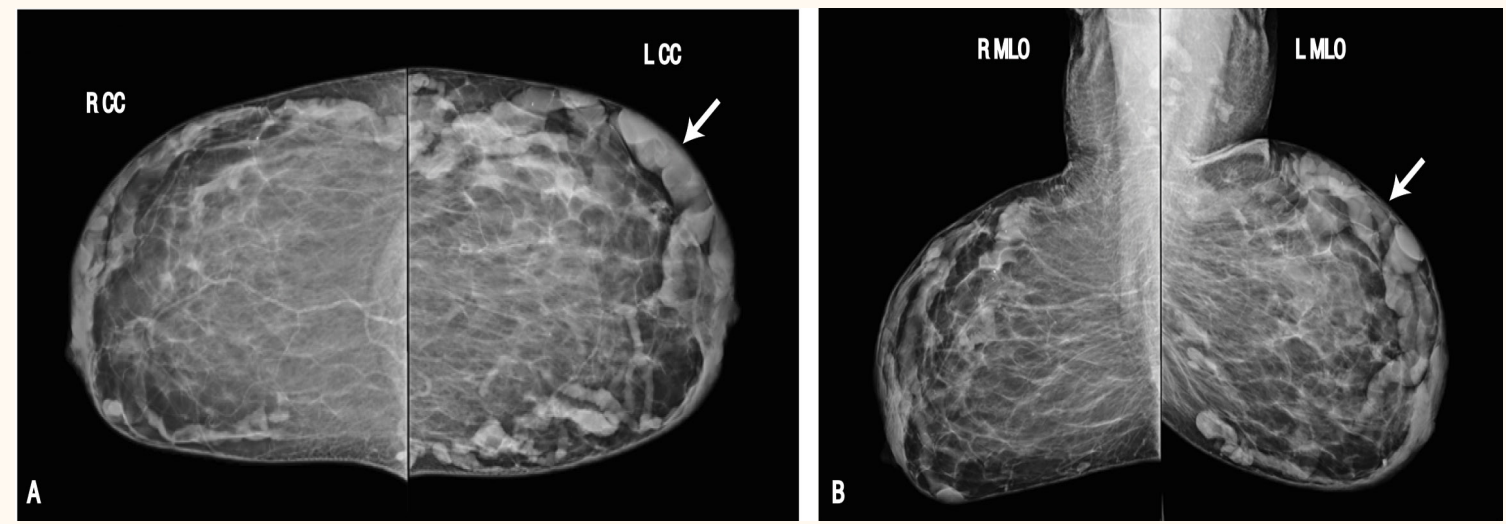

Figure 1: Mammography of both breasts of a 52-year-old postmenopausal female from the (A) craniocaudal and (B) mediolateral oblique aspects showing superficial diffuse cord-like tubular structures (arrows) in the left breast. $R=$ right breast $;=$ left breast $; C C=$ craniocaudal $; M L O=$ mediolateral oblique.

\section{A} 52-YEAR-OLD POSTMENOPAUSAL FEMALE presented to the surgical outpatient department of the Armed Forces Hospital, Muscat, Oman, in 2015 with a mildly tender lump in the left breast of two weeks' duration. She had no associated fever or nipple discharge. The patient had six children whom she had breastfed normally. She was not lactating at the time of presentation and did not have a history of previous breast-related surgeries or trauma. A physical examination revealed a cord-like lump in the lower inner quadrant of the left breast that was mildly tender along with mild erythematic skin changes. The axillary lymph nodes were not palpable.

Mammography and ultrasonography of both breasts was performed. The mammography images showed superficial diffuse cord-like tubular structures in both breasts, although these were more noticeable in the left breast [Figure 1]. In addition, there was evidence of some skin thickening in the areola-nipple complex on the left side. Ultrasonography indicated that the structures were markedly dilated superficial veins.
Some of these veins demonstrated flow on a colour Doppler ultrasound, while a few of the others demonstrated intraluminal thrombosis with no flow [Figure 2].

Based on the imaging findings, a diagnosis of bilateral Mondor's disease was made. The patient was treated symptomatically with anti-inflammatory and analgaesic medications. She responded well to treatment and became clinically asymptomatic six weeks later. At this time, a follow-up scan demonstrated resolution of the superficial venous thrombosis.

\section{Comment}

With an incidence of approximately $0.5-0.8 \%$, Mondor's disease is a very rare benign condition characterised by thrombophlebitis of the subcutaneous veins of the chest wall. ${ }^{1,2}$ The disease is named after Henri Mondor, a French surgeon, who first reported it in 1939. ${ }^{3}$ In most cases, the condition is idiopathic; however, it can be associated with a previous history of breast-related trauma, surgical procedures, biopsies 


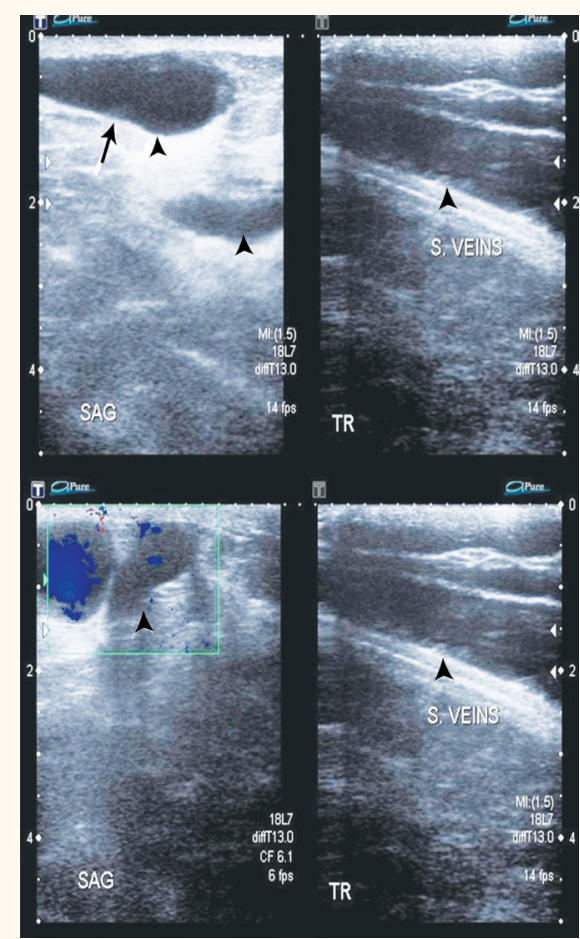

Figure 2: Colour Doppler ultrasonography of both breasts of a 52-year-old postmenopausal female showing flow in some of the superficial veins (arrow) and intraluminal thrombosis with no flow in a few of the others (arrowheads).

$S A G=$ sagittal $S=$ superficial $; R=$ transverse.

or central venous catheter insertion. ${ }^{4}$ A few cases have reported an association with breast cancer, although as yet no direct relationship has been established. ${ }^{1,4}$ In cases where the underlying aetiology is not obvious, patients with Mondor's disease should be investigated to determine the cause of the disease, if possible, and to exclude breast cancer, lymphatic spread from a carcinoma, a hypercoagulable state or connective tissue disease., ${ }^{2,4}$ Recognition and differentiation of this rare condition from a breast abscess or malignancy is also essential. ${ }^{4}$

On mammography, thrombosed veins observed in cases of Mondor's disease are superficial, tubular and beaded cord-like densities which correspond to the clinically palpable and tender cord-like breast lumps. ${ }^{1,2}$ However, it is important to avoid mistaking superficial thrombosed veins for dilated ducts during imaging. ${ }^{1}$ In contrast, the thrombosed veins appear on ultrasonography as superficially located long tubular anechoic non-compressible structures interrupted by narrowed areas, giving them a beaded appearance, most commonly located in the upper outer aspect of the breast, unlike dilated ducts. ${ }^{1}$ Moreover, a thrombosed vein at a periareolar location does not terminate at the areola and bilaterality is rare in Mondor's disease. ${ }^{1}$ In their evaluation of imaging findings in Mondor's disease, Yanik et al. concluded that colour Doppler ultrasonography can help in establishing a correct diagnosis as well as monitoring resolution after treatment. ${ }^{5}$ Similar findings were also noted by Adeniji-Sofoluwe et al. ${ }^{6}$ A clot may be seen as an intraluminal filling defect within the dilated superficial veins with no flow on colour or spectral Doppler studies. ${ }^{1,5,6}$ After a diagnosis of Mondor's disease has been made from initial mammographic and sonographic findings, a short-interval imaging follow-up is recommended within six months to monitor resolution of the condition. ${ }^{1,5}$

Since Mondor's disease is benign and selflimiting, conservative management is recommended. This may include the local application of heat, use of systemic or topical anti-inflammatory and analgaesic medications and support for the affected breast by wearing a well-fitting brassière. ${ }^{1}$ Antibiotics are not required unless there is evidence of infection. In the acute phase of the disease, topical anticoagulants/ antithrombotics can be applied along with oral lowmolecular-weight heparin, which can help to relieve associated symptoms of swelling, pain and redness by decreasing the size of the thrombus., ${ }^{2,4}$

\section{References}

1. Shetty MK, Watson AB. Mondor's disease of the breast: Sonographic and mammographic findings. AJR Am J Roentgenol 2001; 177:893-6. doi: 10.2214/ajr.177.4.1770893.

2. Pasta V, D'Orazi V, Sottile D, Del Vecchio L, Panunzi A, Urciuoli P. Breast Mondor's disease: Diagnosis and management of six new cases of this underestimated pathology. Phlebology 2015; 30:564-8. doi: 10.1177/0268355514553494.

3. Mondor H. [Subcutaneous subacute phlebitis of the anterolateral thoracic wall.] Mem Acad Chir (Paris) 1939; 65:1271-8.

4. Paniagua CT, Negron ZD. Mondor's disease: A case study. J Am Acad Nurse Pract 2010; 22:312-15. doi: 10.1111/j.17457599.2010.00513.x

5. Yanik B, Conkbayir I, Oner O, Hekimoğlu B. Imaging findings in Mondor's disease. J Clin Ultrasound 2003; 31:103-7. doi: 10.1002/jcu.10134.

6. Adeniji-Sofoluwe A, Afolabi O. Mondor's disease: Classical imaging findings in the breast. BMJ Case Rep 2011; 2011: bcr0720114521. doi: 10.1136/bcr.07.2011.4521. 